\title{
RELATIVE DISTRIBUTION OF BREAST ASYMMETRY IN PATIENTS REQUESTING AESTHETIC MAMMAPLASTY
}

\author{
Yolanda Zayakova ${ }^{1}$, Hristo Mihaylov ${ }^{2}$ \\ ${ }^{1}$ Division of Burns, Plastic, Reconstructive and Aesthetic Surgery and \\ ${ }^{2}$ Clinic of General Surgery, Department of Surgery, Naval Hospital of Varna, \\ Military Medical Academy of Sofia
}

\begin{abstract}
PURPOSE: Primary breast asymmetries are quite common. They can affect breast volume and shape, position of inframammary fold, position and size of breast base as well as nipple-areola complex. The aim of this study is to investigate the relative distribution of primary breast asymmetries in patients requesting aesthetic mammaplasty and to analyze the obtained results.

MATERIAL AND METHODS: The study covered 360 female patients at a mean age $31,29 \pm 6,05$ years who were admitted to the hospital for aesthetic breast surgery over a period of $\mathbf{1 0}$ years. Various asymmetries such as in the volume, shape, position and size of the mammary gland base, nipple-areola complex and inframammary fold were described, diagnosed and analyzed. Clinical observation, photo-analysis, anthropomorphic measurements and scanner (in case of skeletal deformities) were applied.

RESULTS: The results showed a total of 241 patients with breast asymmetry or $66,9 \%$ of the cases. Bilateral micromastia was registered as the asymmetry with the highest frequency of occurrence or in $65,6 \%$ of the cases. Asymmetries in the position of the nipples on the vertical axis were found out in $88 \%$ of the women followed by irregularities in the vertical level of the inframammary fold diagnosed in $85,9 \%$ of the patients. Tuberous breast deformity was found in $20,3 \%$ of the patients. More than one sign of asymmetry was observed in $93,8 \%$ of the cases.

CONCLUSION: Breast asymmetry is commonly identified in patients requesting aesthetic mammaplasty. Careful preoperative examination and assessment of these abnormalities underlie the correct planning and ensure the positive outcome of the surgical procedure.
\end{abstract}

Key words: breast asymmetry, bilateral micromastia, nipples, anthropomorphic measurements, aestheic mammaplasty

Address for correspondence:

Yolanda Zayakova, $\mathrm{MD}, \mathrm{PhD}$

Division of Burns, Plastic, Reconstructive and Aesthetic

Surgery, Department of Surgery,

Naval Hospital of Varna, Military Medical Academy of Sofia,

3 Hristo Smirnenski Street,

9010 Varna, Bulgaria

tel.:+359888847611

e-mail:zayakova@yahoo.com

Received: November 26, 2012

Accepted: March 04, 2013

\section{INTRODUCTION}

Breast asymmetries can be either congenital, or acquired. The most common cases of acquired asymmetries are due to surgical procedures, tumours, and injuries. Congenital asymmetries appear during puberty. They affect the breast volume, shape, size and position of the breast base, nipple-areola complex, and inframmary fold (IMF) (1). In 1968, Huston (9) first reported such abnormalities. Later on, in 1971, Radlauer (13) published his research in breast asymmetry and called it 'Amazon syndrome'. The term of 'breast asymmetries' was first introduced 
in 1977 by Edstrom (6) who also noted that different morphological features of the breast might be the cause of these asymmetries.

The aim of this retrospective study is to survey some specific parameters of asymmetry and analyze the frequency of occurrence and type of primary breast asymmetries in the patients requesting aesthetic mammaplasty.

\section{MATERIAL AND METHODS}

The clinical contingent of 360 women was retrospectively examined during a period of 10 years (2002-2011). The average age of the patients was $31,29 \pm 6,05$ years ranging from 18 to 51 . The following indicators for assessment of asymmetries were used: breast volume, shape, position and size of the breast base, nipple-areolar complex and IMF as well as existing deformities of the chest wall and ribs. Clinical observation, photo-analysis, anthropomorphic measurements and scanner (in case of skeletal deformities) were applied in all the patients.

Morphological deviations were established by clinical examination and photo-analysis. The degree of deviation was assessed by bilateral measurements of the following distances: fossa jugularis-nipple (Sn$\mathrm{Ni})$; nipple-IMF (Ni-IMF); nipple-middle line of the sternum (Ni-med); nipple-anterior axillar line-(Nilat), fossa jugularis-lowest point of IMF (Sn-IMF); horizontal and vertical size of the areolar-mammary

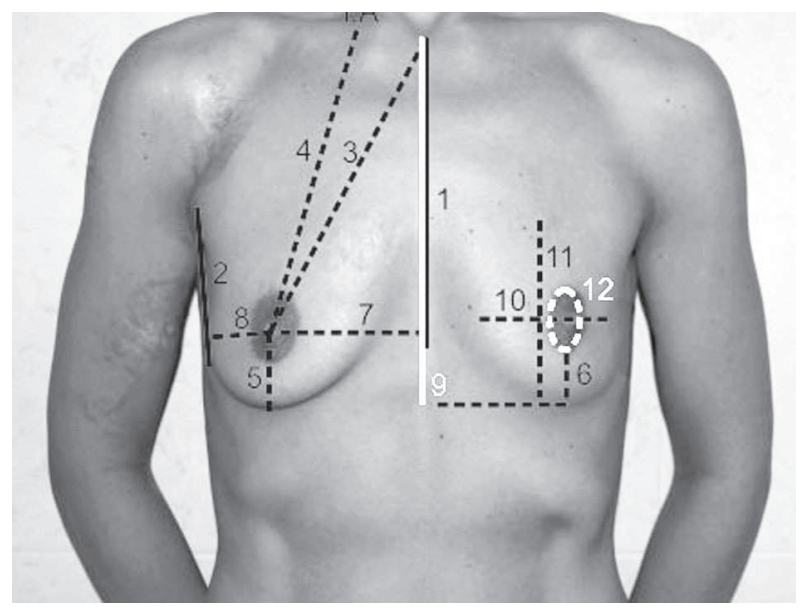

Fig. 1. Antropomorphic measurements

1. Sn-Xy - middle sternal line; 2. Ax-anterior axillary line; 3. Sn-Ni; 4. Ni-IMF; 5. Ni-med; 6. Ni-lat; 7. Sn-IMF; 8. BW; 9. NAC-H; 10. NAC-W complex (NAC-W and NAC-H); horizontal and vertical size of the breast base (BW and BH) (Fig. 1).

The breasts were presumed to be symmetrical if the difference in the measured parameters of both breasts did not exceed $0,5 \mathrm{~cm}$. So, deviations ranging between $0,5-1 \mathrm{~cm}$ were considered slight asymmetry, those between 1-1,5 cm - medium, and those over 1.5 $\mathrm{cm}$ - severe deviation.

Our data was processed by suing SPSS 17.0.1 statistical package. The level of significance was assumed at $\mathrm{p}<0,05$ and the null hypothesis was rejected.

\section{RESULTS}

The antropomorphic measurements revealed breast asymmetries in 241 patients (in $66,9 \%$ of the cases).

Bilateral hypoplasia was the most common breast asymmetry - in 158 patients $(65,6 \%)$ followed by bilateral asymmetric normomasty - in $66(27,4 \%$ of the cases). The unilateral hypoplasia and contralateral hypertrophy occurred most seldom in our contingent - in one case each (only $0,4 \%$ of the cases) (Fig. 2).

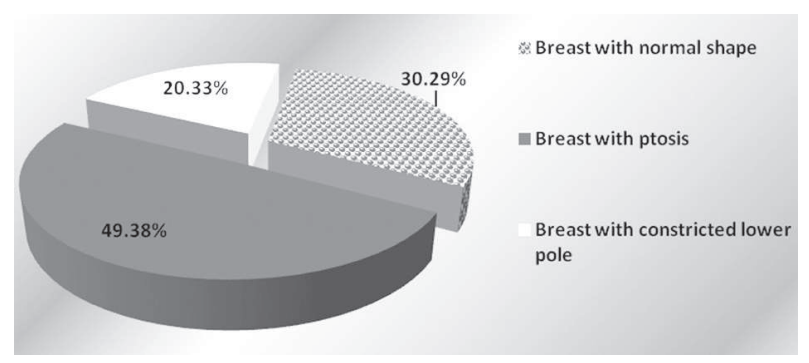

Fig. 2. Distribution of patients with morphological asymmetry

Nipple asymmetry on the vertical axis was the most commonly registered incidence rate - in 212 patients ( $88 \%$ of the cases). Most of them presented with slight asymmetry - in 91 patients $(42,9 \%)$ followed by severe asymmetry - in 61 patients $(28,8 \%)$, and medium one - in 60 cases (28,3\% of the cases).

IMF asymmetry was observed in 207 patients $(85,9 \%)$. The distribution of these cases displayed the same tendency as the abovementioned parameter, namely: from 0,5 to $1,0 \mathrm{~cm}-86$ females $(41,6 \%)$ followed by deviations from 1 to $1,5 \mathrm{~cm}$ - 61 females $(29,5 \%)$. The smallest relative share belonged to the 
patients with asymmetry exceeding $1,5 \mathrm{~cm}-60 \mathrm{fe}-$ males (29\%).

Our study demonstrated that breast base asymmetry was present in $173(71,8 \%)$ cases. More than one half of the patients (101 or $58,4 \%$ ) presented with slight asymmetry followed by those exceeding $1,5 \mathrm{~cm}$ - 41 (23,7\%), and last came those within the range from $1 \mathrm{~cm}$ to $1,5 \mathrm{~cm}$ - 31 cases (17,9\%).

As a whole, cases of asymmetry in the nipple on the horizontal axis were much less observed, more precisely, in 93 out of all the patients $(38,6 \%)$. Slight deviations were identified in 61 of them $(65,6 \%)$, medium asymmetry - in 21 cases $(22,6 \%)$, and severe (over $1,5 \mathrm{~cm}$ ) in 11 cases $(11,8 \%)$.

Patients' distribution according to asymmetry of NAC by size was least in the examined group, more precisely, in 64 patients $(26,6 \%)$ distributed by degree of severity as followed: from 0,5 to $1,0 \mathrm{~cm}-36(56,3 \%)$; from 1 to $1,5 \mathrm{~cm}-16$ (25\%), and those with deviations exceeding $1,5 \mathrm{~cm}-12$ cases (18,8\%) (Fig. 3).

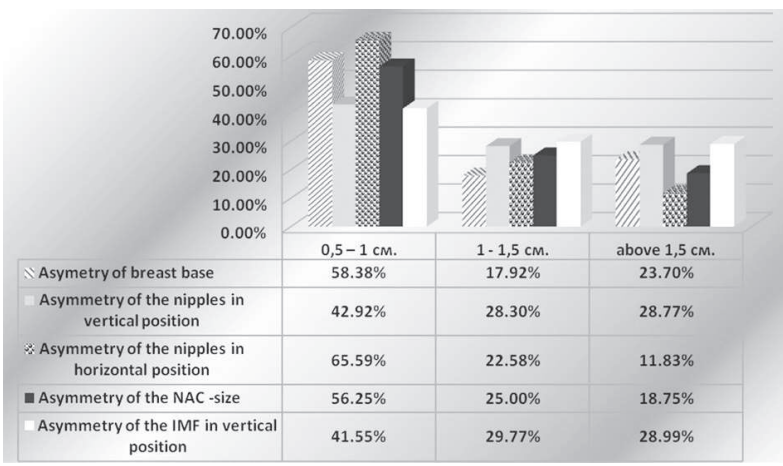

Fig. 3. Patients' distribution according to the level of asymmetry in the position and size of the breast base, $N A C$, nipple and IMF

Concerning breast shape asymmetry, most patients with presented with asymmetry because of ptosis - 119 cases $(49,4 \%)$ followed by those with symmetrical shape - $73(30,3 \%)$. There were only 49

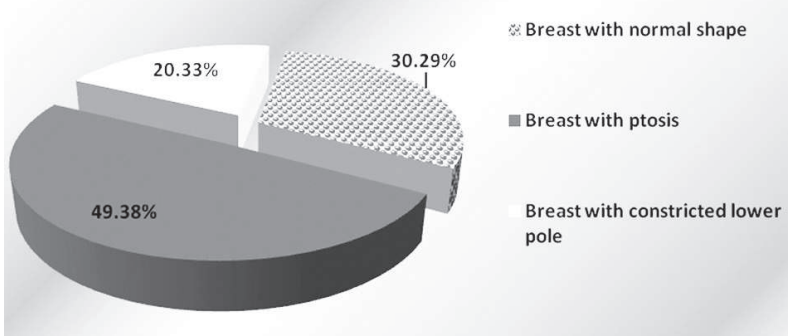

Fig. 4. Patients' distribution according to breast shape patients (20,3\%of the cases) with constriction in the base (Fig. 4).

The distribution of patients with asymmetry and tuberous breast was the following: the highest relative share belonged to type I (13 cases or $41,9 \%)$ followed by type II (5 cases or 16,1\%). Type III and mixed type I+II were equally presented with 4 patients or $12,9 \%$ each. Mixed type II+III was found in two cases $(6,5 \%)$ only (Fig. 5).

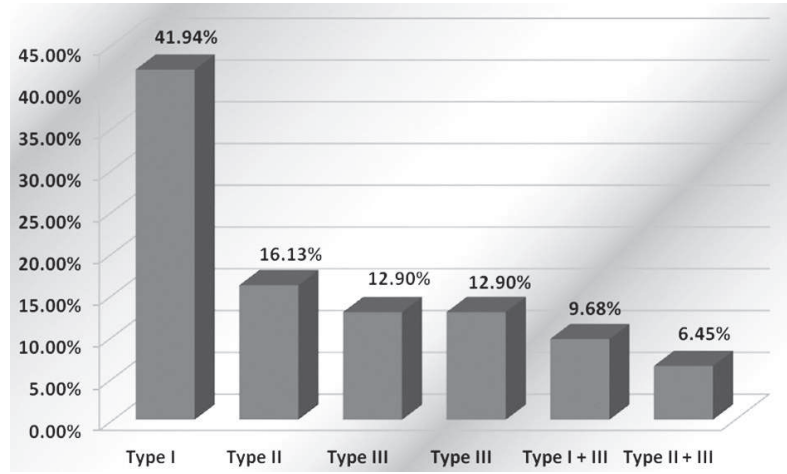

Fig. 5. Patients' distribution according to type of tuberous breast

This deformity was bilateral in $78 \%$ of the patients followed by the females with right breast deformity (in 12\%) and left breast one (in 10\% of the cases).

The prevailing cases of breast asymmetry and tubular deformity were of type II - 12 patients or $66,7 \%$ followed by type I with 6 cases or 33,3\% (Fig. 6).

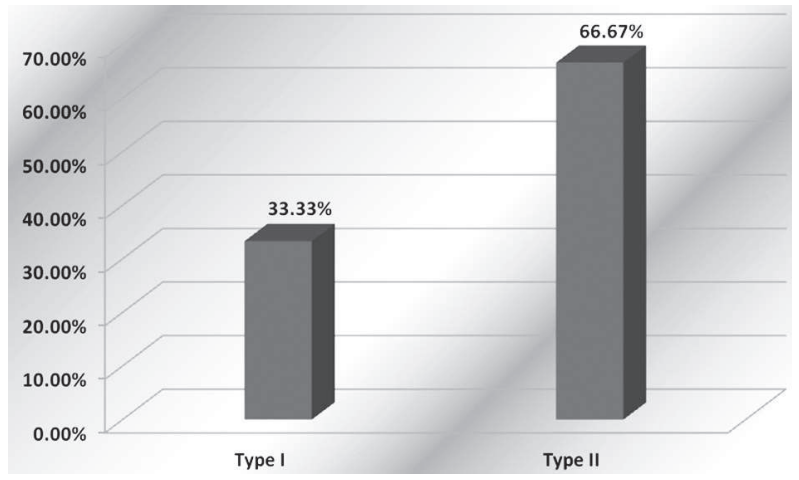

Fig. 6. Patients' distribution according to type of tubular breast

In our study, more than one sign of asymmetry was most commonly observed - in 226 females (in $93,8 \%$ of the cases). Most cases (100 or 41,5\%) showed three signs of asymmetry followed by the patients 
with two signs (50 or $20,7 \%$ ), four signs (46 or $19,1 \%$ ) and five signs (30 or 12,4\%). The smallest number of women presented with one sign of breast asymmetry only - 15 or $6,2 \%$ (Fig. 7).

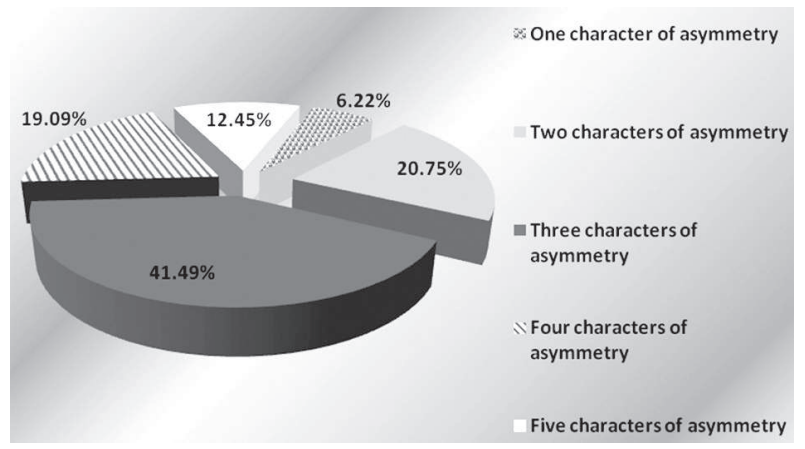

Fig. 7. Patients' distribution according to identified parameters of asymmetry

In most patients (in $91,3 \%$ of the cases), asymmetries were not accompanied by any thoracic deformity at all. Such a combination was established in 21 patients (in $8,7 \%$ of the cases). Six of them $(2,5 \%)$ presented with chest wall depression, four ones $(1,7 \%)$ with prominent rib-sternal junction and pectus excavatum each, and two ones $(0,8 \%)$ - with pectus carinatum (Fig. 8).

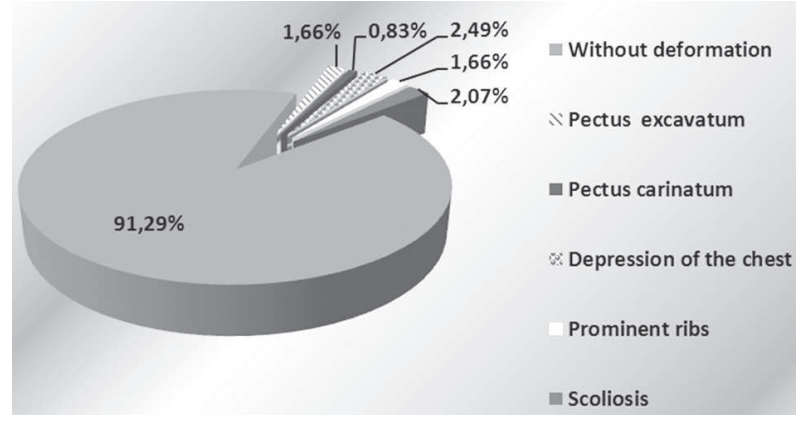

Fig. 8. Distribution of patients with asymmetry and chest deformities

Note: Certain data discrepancies are due to the presence of several indicators of asymmetry in one and the same patient.

\section{DISCUSSION}

Breast asymmetries were defined in terms of asymmetric morphology of volume, shape, position of the base and of the nipple-areolar complex. They were quite common, and in most cases the asymmetries were slight followed by medium. In our contingent of patients, breast asymmetries were established in $66,9 \%$ of the cases. This result were in agreement with the findings of other authors $(5,15)$ but different from those reported by Rohrich (14) about an incidence rate of preoperative asymmetry of $88 \%$. The investigations of the distribution of asymmetries according to their morphological characteristics showed that bilateral micromastia was most common (in 158 females or in $65.6 \%$ of the cases) followed by bilateral asymmetric normomastia (in 66 women or in $27,4 \%$ of the cases).

These findings of ours correspond to the results of other authors $(2,3,6)$. The high incidence rate of micromastia established in patients has to be properly recognized and taken into account while performing augmentation mammaplasty. In such cases if the preoperative assessment does not identify even the slightest irregularity, then the implants may enhance the asymmetry and the result will be far from satisfactory both for the patient and the surgeon. Asymmetry in the position of nipples on the vertical axis has been found out in $88 \%$ of the patients. Our result is similar to the findings already reported by other investigators $(3,10)$. Asymmetric tuberous deformity has been observed in $20,3 \%$ of the clinical contingent. This percentage is significantly less than that of other authors who have identified asymmetries in $81,1 \%$ of these patients as $88,8 \%$ of them are of constricted lower pole (5). Asymmetry of IMF level on the vertical axis is registered in $85,9 \%$ of the patients. Our findings differ greatly from these reported by other authors identifying asymmetry of IMF position in only $44 \%$ of the patients (11). In this contingent, there is more than one indicator of asymmetry in $93,8 \%$, the most common occurrence being three indicators (in 41,5\%). Our own results correlate with the findings about $92 \%$ (11) and about $80 \%$ (12) of the asymmetries with more than one indicator. There is breast asymmetry combined with thoracic deformities in $8,7 \%$ of our cases that corresponds to the results reported by other authors $(7,8)$. Numerous authors perform investigations of breast asymmetries $(1,2,4,10)$. The purpose of their research is to achieve precise preoperative assessment of the breasts as ac- 
Yolanda Zayakova, Hristo Mihaylov

curate estimation of asymmetries is of fundamental significance for the positive postoperative results.

It is a really hard task, if not sometimes an impossible one, to achieve perfect symmetry and proportions. However, the major duty of every surgeon is to make the two breasts look the same. Preoperative morphological measurements allow for mathematically accurate estimations of differences in the position and size of the bases, the nipple-areolar complex and IMF as well. The exact measurements ensure precise surgical correction of all the identified breast asymmetries.

\section{CONCLUSION}

Breast asymmetry occurs quite common among the patients requesting aesthetic mammaplasty. Accurate and objective preoperative assessment and description of the identified deviations contribute significantly to the successful outcome of surgery.

\section{Acknowledgements}

We would like to thank G. Genchev for his assistance in data analyzing as well as P. Dichev and $Y$. Raynov for their technical support in the preparation of the manuscript.

\section{REFERENCES}

1. Araco, A., G. Gravante, F. Araco, P. Gentile, F. Castri, D. Delogu, et al. Breast asymmetries: a brief review and our experience.- Aesth. Plast. Surg., 30, 2006, No 3, 309-319.

2. Bruschi, S., P. Bogetti, M. A. Bocchiotti, N. Kefalas, F. Boriani, D. Marchesi, et al. Congenital mammary asymmetry. Classification and surgical treatment.- Ann. Chir. Plast. Esthet., 78, 2007, No 3, 177-182 (in French).

3. de Chardon, V. M., T. Balaguer, B. Chignon-Sicard, T. Ihrai, E. Lebreton. Constitutional asymmetries in aesthetic breast augmentation: incidence, postoperative satisfaction and surgical options.Ann. Chir. Plast. Esthet., 54, 2009, No 4, 340-347 (in French).

4. Denoel, C., M. F. Aguirre, G. Bianco, P. H. Mahaudens, R. Vanwijck, S. Garson, et al. Idiopathic scoliosis and breast asymmetry.- J. Plast. Reconstr. Aesth. Surg., 62, 2009, No 10, 1303-1308.

5. De Luca-Pytell, D. M., R. C. Piazza, J. C. Holding, N. Snyder, L. M. Hunsicker, L. G. Phillips. The incidence of tuberous breast deformity in asymmet- ric and symmetric mammaplasty patients.- Plast. Reconstr. Surg., 116, 2005, No 7, 1894-1899; discussion, 1900-1901.

6. Edstrom, L. E, M. C. Robson, J. K. Wright. A method for the evaluation of minor degrees of breast asymmetry.- Plast. Reconstr. Surg., 60, 1977, No 5, 812-814.

7. Feng-Chou, T., H. Ming-Shium, L. Chuh-Kai, W. Shu-Ting. Correlation between scoliosis and breast asymmetries in women undergoing augmentation mammaplasty.- Aesth. Plast. Surg., 34, 2010, No 3, 374-380.

8. Gabriel, A., S. Fritzsche, C. Creasman, W. Baqai, D. Mordaunt, G. P. Maxwell. Incidence of breast and chest wall asymmetries: 4D photography.- Aesth. Surg. J., 31, 2011, No 5, 506-510.

9. Hueston, J. T. Surgical correction of breast asymmetry.- Aust. New Zeal. J. Surg., 38, 1968, No 2, 112-116.

10. Khan, U. D. Breast and chest asymmetries: classification and relative distribution of common asymmetries in patients requesting augmentation mammoplasty.- Eur. J. Plast. Surg., 34, 2011, No 5, 375-385.

11. Liu, C., J. Luan, L. Mu, K. Ji. The role of threedimensional scanning technique in involution of breast asymmetry in breast augmentation: A 100 case study.- Plast. Reconstr. Surg., 126, 2010, No 6, 2125-2132.

12. Mugea, T. T. Breast assessment and implant selection using the TTM aesthetic breast chart.- In: Breast augmentation - principles and practice. $\mathrm{M}$. A. Shiffman, ed. Spinger-Verlag, 2008, 185-108.

13. Radlauer, C. B., D. G. Bowers, Jr. Treatment of severe breast asymmetry.- Plast. Reconstr. Surg., 47, 1971, No 4, 347-350.

14. Rohrich, R. J., W. Hartley, S. Brown. Incidence of breast and chest wall asymmetry in breast augmentation: a retrospective analysis of 100 patients.- Plast. Reconstr. Surg., 118, 2006, Suppl. 7, 7S-13S; discussion 14S, 15S-17S.

15. van Aalst, J. A., J. D. Phillips, A. M. Sadove. Pediatric chest wall and breast deformities.- Plast. Reconstr. Surg., 124, 2009, Suppl. 1, 38e-49e. 